\title{
Higiene bucal, caries dental y necesidades de tratamiento en escolares de 5, 7 y 12 años, municipio de Istmina, Chocó, Colombia (2013)
}

Oral Hygiene, Dental Caries and Treatment Needs in 5, 7 and 12 Year-Old School Children in the Village of Istmina, Chocó, Colombia (2013)

Carlos Fernando Hurtado-Martinez

Práctica privada, Medellín, Colombia

carlos-fernando-13@hotmail.com

Dimas Albeiro Potes-Urrutia

Práctica privada, Medellín, Colombia

dimaspotes@hotmail.com

Margarita Vásquez-Díaz

Práctica privada, Medellín, Colombia

mavas_64@hotmail.com

Adriana Posada López

Universidad de Antioquia, Colombia

adriposalo@gmail.com

Luis Gonzalo Álvarez-Sánchez

Universidad de Antioquia, Colombia

lgonzalvarez@gmail.com

Andrés A. Agudelo-Suárez

Universidad de Antioquia, Colombia

oleduga@gmail.com
DOI: https://doi.org/10.11144/Javeriana.uo36-77.hbcd Redalyc: http://www.redalyc.org/articulo.oa? $\mathrm{id}=231254499012$

Fecha de recepción: 25 Abril 2017 Fecha de aprobación: 08 Noviembre 2017

\section{Resumen:}

Antecedentes: Es necesario identificar la situación de salud bucal en grupos específicos para establecer programas efectivos. Objetivo: Describir el estado de higiene bucal, caries dental y de necesidades de tratamiento en escolares de 5, 7 y 12 años del municipio de Istmina (Chocó, Colombia). Métodos: Se realizó un estudio descriptivo en 294 escolares ( 48 \% niñas), seleccionados por conveniencia, de 6 instituciones educativas oficiales. Por medio de encuesta y examen clínico se recogió información sociodemográfica, de uso de elementos de higiene bucal, grado de higiene bucal, índices ceo-d y COP-D (y sus componentes) y necesidades de tratamiento. Se efectuó análisis descriptivo univariado y bivariado e inferencial mediante chi cuadrado. Resultados: Dos terceras partes de la población analizada tenían una higiene oral deficiente (mayor porcentaje en niños y en escolares de 7 años). El promedio ceo-d M fue de 2,5 $\pm 3,2$, lo cual equivale a una prevalencia y experiencia de caries dental del $57 \%$ y $59 \%$ respectivamente. El promedio COP-D M fue de 0,6 $\pm 1,2$, con una prevalencia y experiencia de caries dental del $24 \%$ y $28 \%$ respectivamente. El índice de caries significativa fue de 1,9 $\pm 1,5$. El 47 \% requería obturaciones (promedio de 1,9 $\pm 1,5$ ). Se presentaron diferencias significativas por edad y sexo tanto para caries dental como para necesidades de tratamiento. Conclusiones: Los indicadores de caries dental estaban en consonancia con las metas internacionales. Se identificaron algunas diferencias según factores sociodemográficos que requieren estudios a mayor profundidad desde la perspectiva de determinantes sociales.

Palabras clave: caries dental, Chocó, Colombia, encuestas epidemiológicas, higiene bucal, Istmina, necesidades y demandas de servicios de salud, salud bucal.

Áreas temáticas: epidemiología oral; odontología; salud pública oral 


\begin{abstract}
:
Background: There is a need to identify the oral health situation in every specific group in order to implement effective programs. Objective: To describe the oral hygiene status and treatment needs in 5, 7 and 12 year-old school children in the village of Istmina (Chocó, Colombia). Methods: A descriptive study was carried out in 294 school children ( $48 \%$ girls) selected for convenience reasons from six public schools. Clinical examinations were conducted, and surveys applied in order to gather information on sociodemographic, use of oral hygiene elements, oral hygiene level, ceo-d index and COP-d indexes (and its components), and treatment needs. A descriptive univariate analysis and an inferential bivariate analysis were conducted based on a Chi-square statistics. Results: Two thirds of the studied population had poor oral hygiene habits (a greater percentage was found in 7 year-old boys). The average ceo-d M index was $2.5 \pm 3.2$, which indicates a dental caries prevalence and experience of $57 \%$ and $59 \%$, respectively. The average COP-DM index was $06 \pm 1.2$, with a dental caries prevalence and experience of $24 \%$ and $28 \%$, respectively. The significant dental caries index was $1.9 \pm 1.5 ; 47 \%$ of them required obturations (average: $1.9 \pm 1.5$ ). Signifi cant differences were found as per age and gender, both for dental caries and treatment needs. Conclusions: Dental caries indicators were similar to the expected international goals. Some differences were identified based on some sociodemographic factors, which requires more in-depth studies under a social determinant perspective.
\end{abstract}

Keywords: dental caries, Istmina, Chocó, Colombia, epidemiologic survey, oral hygiene, health care needs and demand, oral health.

Thematic fields: dentistry; oral epidemiology, oral public health

\title{
INTRODUCCIÓN
}

Aunque la epidemiología de las enfermedades bucales es variable (1), continúa considerándose un problema de salud pública que requiere atención (2,3). Deben identificarse la magnitud y la severidad de dichas patologías en los diferentes grupos poblacionales y reconocerse los determinantes sociales que generan situaciones de desigualdad e inequidad (diferencias observables, injustas y evitables) en los indicadores epidemiológicos, a razón de características como sexo, edad, clase social, grupo étnico o territorio $(4,5)$.

En los últimos 50 años, Colombia ha realizado cuatro estudios nacionales de salud bucal $(1966,1980$, 1998, 2013-2014) (6,7,8,9) y una Encuesta Nacional de Salud Pública (2007) (10). Esta última incluyó un componente de percepción de problemas relacionados con la salud bucal y la "Línea de base de seguimiento a metas" del Plan Nacional de Salud Pública 2010 (11), que involucró el análisis de indicadores de caries dental. La meta propuesta dentro de ese plan fue lograr un Índice de Dientes Cariados, Obturados o Perdidos (COP-D) promedio a los 12 años de edad menor de 2,3. Si bien, a grandes rasgos, se evidencia una disminución en la prevalencia de la caries dental y en la enfermedad periodontal, todavía existen situaciones de desigualdad e inequidad en diferentes grupos poblacionales, lo cual sugiere la necesidad de realizar estudios en poblaciones específicas, por ejemplo, en los grupos étnicos minoritarios (especialmente población afrodescendiente). De acuerdo con información disponible en el último censo nacional (2005), el $14 \%$ de la población pertenece a estos grupos y un $11 \%$ se considera afrodescendientes (12).

Por lo tanto, se requiere identificar las necesidades en materia de salud bucal de diferentes grupos poblacionales y sociales. Estudios llevados a cabo en otros países muestran resultados disímiles en la población afrodescendiente. Por un lado, algunos estudios sugieren que esta población tiene mejores indicadores de salud bucal (menor prevalencia de caries dental), teniendo en cuenta su composición microbiana (13). Por otro, algunos estudios muestran peores indicadores en la población afrodescendiente, que se explica por los determinantes sociales, condiciones de vida y dificultades de acceso a los servicios de salud $(14,15)$. En Colombia han sido pocas las investigaciones específicas enfocadas en poblaciones diversas étnica y culturalmente (16). Este estudio parte de un interés por conocer una realidad social en un grupo específico de escolares en el departamento del Chocó en la costa pacífica colombiana, para contribuir a generar estrategias de promoción y prevención, de acuerdo con la situación encontrada, y sensibilizar a los diferentes actores encargados de las políticas y estrategias en salud de la región. Teniendo en cuenta lo anterior, el objetivo de este estudio fue describir el estado de salud bucal y las necesidades de tratamiento en los escolares de 5, 7 y 12 años del área urbana del municipio de Istmina (Chocó). 


\section{MATERIALES Y MÉTODOS}

Se realizó un estudio descriptivo. La población de estudio la constituyeron los escolares de 5, 7 y 12 años de edad matriculados durante 2013 en 6 instituciones educativas oficiales del municipio de Istmina, ubicado en el departamento del Chocó, en la costa pacífica colombiana. Se seleccionaron estas edades trazadoras, en consonancia con las propuestas por el III Estudio Nacional de Salud Bucal (8) -es importante mencionar que durante el desarrollo del trabajo de campo de la presente investigación los resultados del IV estudio nacional no estaban publicados ni disponibles-. Se seleccionaron 294 escolares, mediante un muestreo no probabilístico por conveniencia, teniendo en cuenta características como edad y sexo, y la disponibilidad y aceptación voluntaria de participar en los centros educativos seleccionados para el estudio.

Se diseñó un instrumento para recolectar información adaptado del III Estudio Nacional de Salud Bucal, el cual contenía información sociodemográfica (edad y sexo), uso de elementos de higiene bucal, grado de higiene bucal, estado de la dentición y necesidad de tratamiento. La recolección estuvo a cargo los tres primeros autores del presente artículo (estudiantes de pregrado en formación de investigación), quienes recibieron capacitación y calibración sobre los indicadores de salud bucal e higiene oral (concordancia intra e interobservador: 80 \%). El instrumento de evaluación clínica se ensayó y estandarizó mediante prueba piloto en 20 escolares de la ciudad de Medellín, con características similares a la población de estudio, lo cual permitió revisar la consistencia general y establecer el protocolo de evaluación y diagnóstico de los indicadores propuestos, siguiendo los parámetros de la Organización Mundial de la Salud para encuestas de salud bucodental (17). Esto permitió tener un mayor control de los posibles sesgos que se originaran en el estudio.

Las variables consideradas en este estudio fueron las siguientes:

1) Índice de higiene oral simplificado de Green y Vermillon (IHO-s) (18). Se registró el promedio, que iba en un rango de 0 a 3,0 (a mayor promedio, más presencia de placa).

2) Con la información del IHO-s se obtuvo el grado de higiene oral: bueno (0-1,29), regular, (1,30-3,09) y malo $(>3,10)$.

3) El Índice COP-D de Klein y Palmer (19), para dentición permanente y el Índice ceo-d de Gruebbel para dentición temporal (cariados, obturados y con extracción indicada por caries-por diente) (20). En ambos casos se incluyó la versión tradicional y modificada. La modificación consistió en incluir en el registro de caries las lesiones activas no cavitacionales, de acuerdo con los lineamientos metodológicos de la Línea de Base de Seguimiento a Metas del Plan Nacional de Salud Pública (2010) (11).

4) Índice de Caries Significativa $(\mathrm{SiC})(21)$, que consiste en calcular el promedio COP-D en el tercio más afectado de la población (a partir de la versión tradicional y modificada).

Los datos recolectados se digitalizaron en una hoja de cálculo en Excel y se analizaron con el programa IBM-SPSS, versión 22 para Windows. Se calcularon promedios y desviaciones estándar para todos los índices (COP-D/ceo-d tradicional y modificado, $\mathrm{SiC}$, $\mathrm{IHO}-\mathrm{s}$ ) y las frecuencias absolutas y relativas para: la experiencia y prevalencia de caries dental, el grado de higiene oral a partir del IHOs y las necesidades de tratamiento tanto de forma general como según edad y sexo. Se calcularon pruebas de inferencia estadística tipo chi cuadrado para observar diferencias entre las variables seleccionadas para este estudio.

Esta investigación se rigió bajo los parámetros internacionales (22) y nacionales para estudios en seres humanos (23). Según la normativa para Colombia, se considera una investigación de riesgo mínimo (23). Se garantizó la confidencialidad durante todo el proceso de investigación, tanto en la base de datos como en la evaluación clínica. Se manejaron los protocolos de examen odontológico y normas de bioseguridad para los participantes. Se obtuvo el consentimiento informado para participar por parte de los padres de familia y acudientes de los escolares y para la realización del examen. De igual forma, se contó con la aprobación de las directivas de las instituciones educativas del municipio de Istmina. Esta investigación se aprobó como un ejercicio académico de investigación realizado por tres de los autores en el pregrado de la 
Facultad de Odontología de la Universidad de Antioquia, Medellín. Este artículo tuvo en cuenta las pautas internacionales para el reporte de estudios observacionales en epidemiología (Pauta STROBE, por su sigla en inglés) (24).

\section{RESULTADOS}

Se evaluaron 294 escolares de seis instituciones educativas, con mayor frecuencia del sexo masculino, en el 52 $\%$ de los casos. Las edades de los escolares se distribuyeron de menor a mayor frecuencia, así: 11,2 \%, 39,5\% y 49,3\%, para los de 5, 12 y 7 años, respectivamente. Se indagó en esta población el uso de implementos de higiene bucal, sobresaliendo el uso del cepillo (99\%) y la crema dental (98\%), sin diferencias estadísticamente significativas según edad y sexo. El $10 \%$ de los escolares evaluados usaban enjuague bucal (mayor proporción en niños de sexo masculino y en el grupo de edad de 12 años fue similar en ambos sexos). El uso de la seda dental se reportó en el $3 \%$ (mayor proporción en niñas y en el grupo de edad de 12 años fue igual para ambos sexos). El uso de otros implementos de higiene bucal como palillos, bicarbonato, cenizas, sal y hierbas se reportó esporádicamente (porcentajes menores al 1,5\%).

En cuanto al grado de higiene oral IHO-s (tabla 1), tanto el promedio como la mediana son más altos en población escolar de sexo masculino y en niños y niñas de 7 años. Esto representa que el $68 \%$ de los niños y el $72 \%$ de los escolares de 7 años tienen un grado de higiene oral regular a partir de la clasificación cualitativa del IHO-s (tabla 2).

TABLA 1

Índice de Higiene Oral Simplificado (IHO-s) en los escolares de Istmina (Chocó, Colombia) según edad y sexo*

* IC95 \%: Intervalo de confianza al $95 \%$. RIC: Rango Intercuartílico.

\begin{tabular}{lcccc}
\hline \multirow{2}{*}{ Variables } & \multicolumn{5}{c}{ IHO-s } \\
\cline { 2 - 5 } & Promedio & IC $95 \%$ & Mediana & RIC \\
\hline Sexo & & & 1,50 & $1,00-1,83$ \\
$\quad$ Hombre & 1,60 & $1,39-1,81$ & 1,50 & $1,00-1,83$ \\
$\quad$ Mujer & 1,48 & $1,38-1,83$ & & \\
Edad & & & 1,66 & $1,00-2,17$ \\
5 & 1,64 & $1,41-1,87$ & 1,50 & $1,16-1,83$ \\
7 & 1,67 & $1,44-1,89$ & 1,83 & $1,00-1,66$ \\
12 & 1,36 & $1,27-1,46$ & 1,33 & $\mathbf{1 , 0 0 - 1 , 8 3}$ \\
Total & $\mathbf{1 , 5 4}$ & $\mathbf{1 , 4 2 - 1 , 6 6}$ & $\mathbf{1 , 5 0}$ & $\mathbf{1 , 0 6}$ \\
\hline
\end{tabular}

TABLA 2

Grado de higiene oral en los escolares de Istmina (Chocó, Colombia) según edad y sexo

\begin{tabular}{ccc}
\hline \multirow{2}{*}{ Variables } & \multicolumn{2}{c}{$\begin{array}{c}\text { Grado de higiene oral } \\
\text { (\%) }\end{array}$} \\
\cline { 2 - 3 } Sexo & Bueno & Regular \\
\hline Hombre & 32,0 & 68,0 \\
$\quad$ Mujer & 34,8 & 65,2 \\
Edad & & \\
5 & 36,4 & 63,6 \\
7 & 27,6 & 72,4 \\
12 & 39,7 & 60,3 \\
Total & $\mathbf{3 3 , 3}$ & $\mathbf{6 6 , 7}$ \\
\hline
\end{tabular}


En la tabla 3 se muestran los diferentes componentes del índice ceo-d en su versión tradicional y modificada, así como la prevalencia y experiencia de caries dental en dentición primaria. Tanto el promedio de dientes cariados deciduos es mayor a la edad de 5 años (con diferencias significativas para el caso de dientes cavitacionales con respecto a los 7 años) y en niños (al igual que la prevalencia de caries dental en ambos casos). La prevalencia de caries dental en dentición primaria fue del $57 \%$, al tener en cuenta todas las lesiones cariosas y del $52 \%$ solo con las lesiones cavitacionales (lo cual representa una experiencia del $55 \%$ y $59 \%$, respectivamente). Se presentó un bajo promedio de dientes obturados y con extracción indicada por caries en ambas edades y sexos.

TABLA 3

Promedio (X) y desviación estándar (DE) del ceo-d tradicional, modificado y sus respectivos componentes en los escolares de Istmina (Chocó, Colombia) según edad y sexo c1: cariados deciduos no cavitacionales; c2: cariados deciduos cavitacionales; ceo-d T : índice ceo tradicional; ceo-dM: índice ceo modi有 cado. PrevalenciaT-PrevalenciaM: Prevalencia calculada según c1 > 0 y c1 $+\mathrm{c} 2>0$, respectivamente (\%). ExperienciaT-ExperienciaM: Experiencia calculada según ceo-dT $>0 \mathrm{y}$ ceo-dM $>0$ respectivamente $(\%)$. Pruebas estadísticas inferenciales: ${ }^{*} \mathrm{p}<0,05 ;{ }^{* *} \mathrm{p}<0,01 ;{ }^{* * *} \mathrm{p}<0,001$

\begin{tabular}{|c|c|c|c|c|c|}
\hline Variables & $\begin{array}{c}5 \text { años } \\
(n=33)\end{array}$ & $\begin{array}{c}7 \text { años } \\
(n=145)\end{array}$ & $\begin{array}{c}\text { Niños } \\
(\mathrm{n}=\mathbf{9 8})\end{array}$ & $\begin{array}{c}\text { Niñas } \\
(n=80)\end{array}$ & $\begin{array}{c}\text { Total } \\
(n=178)\end{array}$ \\
\hline Sanos & $14,2 \pm 4,1^{* *}$ & $11,7 \pm 4,0^{* *}$ & $11,9 \pm 4,1$ & $12,6 \pm 4,1$ & $12,2 \pm 4,1$ \\
\hline $\mathrm{cl}$ & $0,3 \pm 1,1$ & $0,3 \pm 0,8$ & $0,3 \pm 0,9$ & $0,3 \pm 0,8$ & $0,3 \pm 0,9$ \\
\hline $\mathrm{c} 2$ & $3,0 \pm 3,5^{* *}$ & $1,7 \pm 2,4^{* *}$ & $2,1 \pm 2,3$ & $1,7 \pm 2,4$ & $1,9 \pm 2,7$ \\
\hline $\mathrm{c} 1+\mathrm{c} 2$ & $3,3 \pm 3,6^{*}$ & $1,9 \pm 2,8^{*}$ & $2,4 \pm 3,1$ & $1,9 \pm 2,8$ & $2,2 \pm 3,0$ \\
\hline $\mathrm{O}$ & $0,1 \pm 0,2$ & $0,1 \pm 0,4$ & $0,1 \pm 0,5$ & $0,1 \pm 0,2$ & $0,1 \pm 0,4$ \\
\hline e & $0,0 \pm 0,0^{*}$ & $0,2 \pm 0,6^{*}$ & $0,2 \pm 0,6$ & $0,2 \pm 0,6$ & $0,2 \pm 0,6$ \\
\hline ceo-d $\mathrm{d}_{\mathrm{T}}$ & $3,1 \pm 3,6$ & $2,0 \pm 2,7$ & $2,4 \pm 3,0$ & $1,9 \pm 2,8$ & $2,2 \pm 2,9$ \\
\hline ceo-d $\mathrm{d}_{\mathrm{M}}$ & $3,4 \pm 3,7$ & $2,3 \pm 3,1$ & $2,7 \pm 3,3$ & $2,2 \pm 3,2$ & $2,5 \pm 3,2$ \\
\hline Prevalencia & 57,6 & 50,3 & 56,1 & 46,3 & 51,7 \\
\hline Prevalencia & 63,6 & 55,2 & 62,3 & 50,0 & 56,7 \\
\hline Experiencia $_{\mathrm{T}}$ & 57,6 & 54,5 & 59,2 & 50,0 & 55,1 \\
\hline Experiencia & 63,6 & 57,9 & 64,3 & 52,5 & 59,0 \\
\hline
\end{tabular}

En cuanto los componentes del COP-D en su versión tradicional y modificada para la dentición permanente a los 7 y 12 años (tabla 4), un poco menos de la tercera parte de la población estudiada presentó caries dental, teniendo en cuenta todas las lesiones cariosas. La prevalencia de caries dental, considerando las lesiones cavitacionales y todas las lesiones, fue mayor para las niñas y para la población escolar de 12 años (más del $40 \%$ en ambos casos). Se presentaron promedios inferiores a 1,0 diente cariado para ambas edades y sexos, aunque el $\mathrm{SiC}$ aumentaba cuando se tuvieron en cuenta todas las lesiones cariosas (mayor en niñas y en escolares de 12 años). Se presentaron diferencias estadísticamente significativas por edad para todos los promedios de los componentes analizados con excepción de los dientes cariados no cavitacionales. Asimismo, se presentaron diferencias estadísticamente significativas por sexo para el caso del promedio de dientes obturados (mayor en niñas). 
TABLA 4

Promedio $(X)$ y desviación estándar (DE) del COPD tradicional, modificado y sus respectivos componentes en los escolares de Istmina (Chocó, Colombia) según edad y sexo

$\mathrm{C} 1$ : cariados deciduos no cavitacionales; $\mathrm{C} 2$ : cariados deciduos cavitacionales; $\mathrm{COPD}$ : índice COPD tradicional; COPDM: índice COPD modificado. PrevalenciaT-Prevalencia $\mathrm{M}$ : Prevalencia calculada según C1 $>0$ y C1 + C2 >0, respectivamente (\%). ExperienciaT-ExperienciaM: Experiencia calculada según COPDT $>$ 0 y COPD $\mathrm{M}>0$ respectivamente (\%). Pruebas de significación estadística: ${ }^{*} \mathrm{p}<0,05 ;{ }^{* *} \mathrm{p}<0,01 ;{ }^{* *} \mathrm{p}<0,001$.

\begin{tabular}{|c|c|c|c|c|c|}
\hline Variables & $\begin{array}{c}7 \text { años } \\
(\mathrm{n}=145)\end{array}$ & $\begin{array}{c}12 \text { años } \\
(n=116)\end{array}$ & $\begin{array}{c}\text { Niños } \\
(\mathrm{n}=\mathbf{1 3 3})\end{array}$ & $\begin{array}{c}\text { Niñas } \\
(\mathrm{n}=129)\end{array}$ & $\begin{array}{c}\text { Total } \\
(\mathrm{n}=\mathbf{2 6 1})\end{array}$ \\
\hline Sanos & $8,1 \pm 3,1 * * *$ & $24,2 \pm 4,7^{* * *}$ & $14,7 \pm 8,8$ & $15,9 \pm 9,0$ & $15,3 \pm 8,9$ \\
\hline $\mathrm{C} 1$ & $0,1 \pm 0,5$ & $0,2 \pm 0,9$ & $0,2 \pm 0,8$ & $0,1 \pm 0,5$ & $0,2 \pm 0,7$ \\
\hline $\mathrm{C} 2$ & $0,1 \pm 0,5^{* * *}$ & $0,6 \pm 1,1^{* * *}$ & $0,4 \pm 0,9$ & $0,4 \pm 0,8$ & $0,4 \pm 0,8$ \\
\hline $\mathrm{C} 1+\mathrm{C} 2$ & $0,3 \pm 0,8 * * *$ & $0,8 \pm 1,3^{* * *}$ & $0,6 \pm 1,2$ & $0,5 \pm 1,0$ & $0,5 \pm 1,1$ \\
\hline $\mathrm{O}$ & $0,0 \pm 0,2 * *$ & $0,2 \pm 0,6^{* *}$ & $0,0 \pm 0,2 * *$ & $0,2 \pm 0,6^{* *}$ & $0,1 \pm 0,5$ \\
\hline $\mathrm{P}$ & $0,0 \pm 0,0^{* *}$ & $0,1 \pm 0,4 * *$ & $0,0 \pm 0,3$ & $0,0 \pm 0,2$ & $0,0 \pm 0,3$ \\
\hline COP-D & $0,2 \pm 0,6^{* * *}$ & $0,9 \pm 1,3^{* * *}$ & $0,4 \pm 0,9$ & $0,6 \pm 1,1$ & $0,5 \pm 1,0$ \\
\hline COP-DM & $0,3 \pm 0,8 * * *$ & $1,1 \pm 1,5^{* * *}$ & $0,6 \pm 1,3$ & $0,7 \pm 1,2$ & $0,6 \pm 1,2$ \\
\hline $\mathrm{SiC}_{\mathrm{T}}$ & $0,5 \pm 0,9 * * *$ & $2,3 \pm 1,1^{* * *}$ & $1,2 \pm 1,3$ & $1,6 \pm 1,3$ & $1,4 \pm 1,3$ \\
\hline $\mathrm{SiC}_{\mathrm{M}}$ & $0,8 \pm 1,3 * * *$ & $2,8 \pm 1,3^{* * *}$ & $1,8 \pm 1,6$ & $1,9 \pm 1,3$ & $1,9 \pm 1,5$ \\
\hline Prevalencia & $9,0 * * *$ & $32,8^{* * *}$ & 17,4 & 21,7 & 19,5 \\
\hline Prevalencia & $11,7 * * *$ & $39,7 * * *$ & 22,7 & 25,6 & 24,1 \\
\hline Experiencia $\mathrm{T}$ & $10,3 * * *$ & $41,4^{* * *}$ & 19,7 & 28,7 & 24,1 \\
\hline Experiencia & $13,1 * * *$ & $47,4^{* * *}$ & 24,2 & 32,6 & 28,4 \\
\hline
\end{tabular}

Las necesidades de tratamiento en la población estudiada se resumen en las tablas 5 y 6 , de donde sobresale que más de la mitad de la población escolar de 5 y 7 años y casi una tercera parte en los de 12 años requerían obturaciones. En cuanto a la magnitud, los niños y niñas de 5 años necesitaban en promedio 3 obturaciones que disminuían con la edad. Las niñas requerían mayor porcentaje y promedio de sellantes que los niños. Las necesidades en endodoncia no superaban el $3 \%$ para todos los casos. En cuanto a las extracciones, un $7 \%$ del total de escolares evaluados las necesitaban.

TABLA 5

Promedio $(X)$ y desviación estándar (DE) de dientes con necesidades de tratamiento en los escolares de Istmina (Chocó, Colombia) según edad y sexo Pruebas de significación estadística: ${ }^{*} \mathrm{p}<0,05 ;{ }^{* *} \mathrm{p}<0,01 ;{ }^{* *} \mathrm{p}<0,001$.

\begin{tabular}{|c|c|c|c|c|c|c|}
\hline $\begin{array}{c}\text { Necesidades de } \\
\text { tratamiento }\end{array}$ & $\begin{array}{c}5 \text { años } \\
(\mathrm{n}=\mathbf{3 3})\end{array}$ & $\begin{array}{c}7 \text { años } \\
(n=145)\end{array}$ & $\begin{array}{c}12 \text { años } \\
(\mathrm{n}=116)\end{array}$ & $\begin{array}{c}\text { Niños } \\
(\mathbf{n}=\mathbf{1 5 3})\end{array}$ & $\begin{array}{c}\text { Niñas } \\
(\mathrm{n}=141)\end{array}$ & $\begin{array}{c}\text { Total } \\
(\mathrm{n}=\mathbf{2 9 4})\end{array}$ \\
\hline Sellantes & $\begin{array}{c}1,1 \pm \\
1,7^{* * *}\end{array}$ & $\begin{array}{c}2,5 \pm \\
2,7^{* * *}\end{array}$ & $\begin{array}{c}3,3 \pm \\
3,1^{* * *}\end{array}$ & $2,2 \pm 2,7^{*}$ & $3,1 \pm 3,0^{*}$ & $2,6 \pm 2,9$ \\
\hline Obturaciones & $\begin{array}{c}2,9 \pm \\
3,3^{* * *}\end{array}$ & $\begin{array}{c}1,7 \pm \\
2,2 * * *\end{array}$ & $\begin{array}{c}0,6 \pm \\
1,1^{* * *}\end{array}$ & $1,6 \pm 2,3$ & $1,2 \pm 1,9$ & $1,4 \pm 2,1$ \\
\hline Endodoncia-restauración & $0,0 \pm 0,2$ & $0,0 \pm 0,2$ & $0,0 \pm 0,2$ & $0,0 \pm 0,2$ & $0,0 \pm 0,3$ & $0,0 \pm 0,2$ \\
\hline Extracción & $0,2 \pm 0,6$ & $0,1 \pm 0,5$ & $0,1 \pm 0,3$ & $0,1 \pm 0,5$ & $0,1 \pm 0,4$ & $0,1 \pm 0,5$ \\
\hline
\end{tabular}


TABLA 6

Distribución porcentual de necesidades de tratamiento en los escolares de Istmina (Chocó, Colombia) según edad y sexo Pruebas estadísticas inferenciales: ${ }^{*} \mathrm{p}<0,05 ;{ }^{* *} \mathrm{p}<0,01 ;{ }^{* * *} \mathrm{p}<0,001$.

\begin{tabular}{lcccccc}
\hline $\begin{array}{c}\text { Necesidades de } \\
\text { tratamiento }\end{array}$ & $\begin{array}{c}\mathbf{5} \text { años } \\
(\mathbf{n}=\mathbf{3 3}) \\
\mathbf{\%}\end{array}$ & $\begin{array}{c}\mathbf{7} \text { años } \\
(\mathbf{n}=\mathbf{1 4 5}) \\
\mathbf{\%}\end{array}$ & $\begin{array}{c}\mathbf{1 2} \text { años } \\
(\mathbf{n}=\mathbf{1 1 6}) \\
\mathbf{\%}\end{array}$ & $\begin{array}{c}\text { Niños } \\
(\mathbf{n}=\mathbf{1 5 3}) \\
\mathbf{\%}\end{array}$ & $\begin{array}{c}\text { Niñas } \\
(\mathbf{n}=\mathbf{1 4 1}) \\
\mathbf{\%}\end{array}$ & $\begin{array}{c}\text { Total } \\
(\mathbf{n}=\mathbf{2 9 4}) \\
\mathbf{\%}\end{array}$ \\
\hline Sellantes & $33,3^{* *}$ & $60,7^{* *}$ & $63,8^{* *}$ & $52,9^{*}$ & $65,2^{*}$ & 58,8 \\
Obturaciones & $57,6^{* * *}$ & $57,2^{* * *}$ & $31,9^{* * *}$ & 50,3 & 44,0 & 47,3 \\
Endodoncia-restauración & 3,0 & 2,8 & 2,6 & 2,6 & 2,8 & 2,7 \\
Extracción & 6,1 & 8,3 & 5,2 & 5,9 & 7,8 & 6,8 \\
\hline
\end{tabular}

\section{DISCUSIÓN}

Los hallazgos principales de este estudio dan cuenta de cómo en la población escolar del municipio de Istmina se observaron grados de higiene oral regulares, dato que no concuerda con la alta proporción de utilización de cepillo y crema dental, pero hay mayor correspondencia con la baja utilización de seda dental y otros implementos. Se observaron indicadores de caries dental más altos en dentición decidua en comparación con dentición permanente y diferencias por sexo (la prevalencia de caries dental fue mayor en niños en dentición decidua y mayor en niñas en dentición permanente). Una buena parte de las necesidades de tratamiento para esta población correspondía a actividades preventivas, sellantes y obturaciones de menor complejidad, en términos del número de superficies. Hasta donde alcanza nuestro conocimiento, este es uno de los primeros estudios realizados en población escolar del municipio de Istmina en población afrodescendiente.

En cuanto a la utilización de implementos de higiene bucal en la población de estudio, los resultados indicaron que el uso del cepillo de dientes fue generalizado, caso contrario al uso que tuvo la seda dental, en la que se observó un bajo porcentaje. Lo anterior concuerda con los hallazgos de los dos últimos Estudios Nacionales de Salud Bucal realizados en Colombia $(8,9)$, y a manera de ejemplo con otro estudio en población escolar realizado en el área metropolitana de la ciudad de Medellín (25), donde se observó que la mayoría de los niños evaluados tenía el hábito de cepillar sus dientes tres veces al día, y utilizaba el cepillo de dientes como el principal implemento de higiene bucal.

Se encontraron algunas diferencias en cuanto al grado de higiene bucal por sexo y edad. En el presente estudio, el mayor promedio de placa dental se registró en los niños de 7 años y, en general, un grado de higiene oral regular. Estos hallazgos sugieren la necesidad de implementar programas de intervención en el cuidado de la salud y la prevención de las enfermedades bucales, así como hacer hincapié en la salud familiar 
en la que padres y cuidadores realicen la debida supervisión y educación. Lo anterior es sustentado en el estudio de Díaz y colaboradores (26), de 2014, en el que se presenta el modelo de salud familiar y que tiene en su concepción a los individuos como seres integrales que actúan con su entorno. Ese estudio entiende la salud familiar como la relación individuo-familia-sociedad.

El grado de higiene bucal en los escolares evaluados en Istmina fue similar al de un estudio realizado en el municipio de San Francisco, Antioquia, en el que predominó la higiene oral inadecuada (27). Ambos municipios se han catalogado como vulnerables, dado el desplazamiento de la población, bajas condiciones de vida, pobreza y desigualdades sociales. Como lo menciona César Abadía (28), en 2006, quienes viven en pobreza son más vulnerables a padecer enfermedades y a que estas se agraven, para lo cual propone la generación de sistemas de equidad sin disparidades en el diagnóstico y en el tratamiento.

Respecto al índice de caries en dentición decidua, un poco más de la mitad de los hombres y de todos los escolares evaluados a la edad de 5 años estaban afectados por los dos tipos de lesiones, de mancha blanca y cavitacionales. La edad de los cinco años es pertinente para realizar comparaciones internacionales, en cuanto a la situación de salud bucal en dentición primaria, el cual fue más bajo en los niños de Istmina, que en lo reportado en el ENSAB IV (88 \%) (9). Los resultados son inferiores a los del estudio sobre morbilidad oral realizado en 110 niños escolares de una población de indígenas amazónicos, en los que se encontró una prevalencia de caries en dientes temporales del 62,6\%.

En este estudio, la prevalencia de caries dental, al tener en cuenta todos los tipos de lesiones, fue mayor en las niñas de 12 años (40\%); mientras que para los niños de la etnia de la Amazonia fue del 28,8 \%, con un $\mathrm{SiC}$ de 2,08 (29). Es importante tener en cuenta que se comparan los resultados con otras etnias minoritarias ante la dificultad de encontrar estudios específicos en población afrodescendiente escolar en Colombia.

En un estudio en adolescentes en el departamento del Valle (Colombia) se encontró la prevalencia más baja de caries en personas entre 12 y 13 años (65,6\%), pero notaron que los adolescentes el 76,1 \% habían tenido experiencia de caries, lo que posiblemente indica la falta de acceso a los servicios de salud desde la infancia temprana en las diferentes regiones de Colombia (30). Esta situación se confirma con un estudio con datos poblacionales que han mostrado cómo la población de grupos étnicos minoritarios con edades similares a los de la población participante en el estudio de Istmina utilizaban con menor frecuencia los servicios de salud, y esto sugiere algunas situaciones de inequidad y desigualdad en el acceso a los servicios de salud bucal (31). No obstante, en el análisis de las necesidades de tratamiento, más de la mitad de la población estudiada requería obturaciones y, en general, eran de menor complejidad.

La situación de salud bucal va más allá del análisis de los factores individuales y biológicos. Se debe reconocer el contexto social donde se ubica la población escolar seleccionada en el estudio. El municipio de Istmina, ubicado a 74 kilómetros de Quibdó (capital del departamento de Chocó), se encuentra en una zona definida por la Organización de las Naciones Unidas como vulnerable (32), dado que es uno de los departamentos de Colombia donde se ha reportado mayor frecuencia de casos de violación de derechos humanos, pobreza extrema, lucha territorial por parte de diferentes actores y alto número de personas desplazadas, lo que ocasiona un déficit en la seguridad alimentaria, baja calidad de la educación y deficiencias en el sistema de salud, entre otros (33). Lo anterior sugiere que la intervención que requiere dicha población en cuanto a la salud bucal debe superar las barreras de la inequidad, reto que si se alcanzara se retribuiría en mejores condiciones de vida en la población estudiada (34).

Es importante conocer las fortalezas y limitaciones de este estudio. Esta investigación es una de las primeras realizadas en población afrodescendiente escolar en esta zona de Colombia. La información obtenida sirve como línea de base para crear acciones educativas y preventivas por parte de las entidades de salud, a fin de mejorar la condición de salud bucal en esta etnia en la que se tenga como prioridad la formación de personas líderes, profesores y cuidadores que promuevan el respeto por las costumbres y la cultura relacionado con el tema del autocuidado. Como limitaciones, es relevante mencionar que los resultados no se pueden extrapolar a población general, debido al tipo de muestreo empleado. Por otra parte, la naturaleza transversal del estudio 
no permite establecer relaciones causales. Por último, los resultados encontrados deben interpretarse con cautela, ya que los indicadores utilizados para evaluar caries dental (COP-D y ceo-d en su versión tradicional y modificada) presentan dificultades en su comparabilidad con los utilizados en el ENSAB IV (Sistema ICDAS-Epi) (9).

\section{CONCLUSIONES Y RECOMENDACIONES}

Se debe reconocer que la caries dental es una enfermedad crónica y multifactorial compleja; por lo tanto, las políticas y programas que se deben generar en dicho territorio deben enfocarse en las particularidades de la etnia y los modos de vida de la comunidad y familias en los que los niños crecen, sin olvidar la organización cultural que ellos hayan determinado $(35,36)$. Con el fin de incrementar la calidad de vida de las personas y sus familias, se debería fortalecer las capacidades de los líderes comunitarios, profesores de escuelas y colegios y cuidadores. Esto conduciría a la concientización de las personas al autocuidado como factor protector para la salud (37).

De igual forma, se requiere fortalecer los sistemas de vigilancia epidemiológica de las enfermedades bucales, con especial interés en variables que permitan identificar situaciones de desigualdad e inequidad social en grupos especialmente vulnerables. De igual forma, continuar fortaleciendo la investigación en epidemiología bucal desde una perspectiva social e investigar sobre la construcción social de los principales problemas en salud bucal, a partir de estudios cualitativos.

\section{AGRADECIMIENTOS}

El grupo de investigadores agradece la participación y colaboración de los niños y niñas incluidos en el estudio, así como de sus padres y acudientes. Sus aportes constituyen un insumo para la visibilidad de una realidad poco conocida en el país, así como para la generación de estrategias locales en promoción de la salud bucal, basadas en las propias realidades de la comunidad.

Conflicto de intereses: ninguno.

Financiación: recursos propios.

\section{REFERENCIAS}

1. Petersen PE. The World Oral Health Report 2003: continuous improvement of oral health in the 21st century--the approach of the WHO Global Oral Health Programme. Community Dent Oral Epidemiol. 2003; 31(Suppl 1): 3-23. https://doi.org/10.1046/j..2003.com122.x

2. Petersen PE, Bourgeois D, Ogawa H, Estupinan-Day S, Ndiaye C. The global burden of oral diseases and risks to oral health. Bull World Health Organ. 2005; 83(9): 661-9.

3. Escobar P, Ortiz Z, Cecilia A, Mejía O, Mery L. Caries dental en los menores de veinte años en Colombia: un problema de salud pública. Rev Fac Nac Salud Pública. 2003; 21(2): 107-18.

4. Agudelo Suárez AA, Martínez Herrera E. La salud bucal colectiva y el contexto colombiano: un análisis crítico. Rev Gerenc Polit Salud. 2009; 8(16): 91-105.

5. Watt RG. Social determinants of oral health inequalities: implications for action. Community Dent Oral Epidemiol. 2012; 40 Suppl 2: 44-8. https://doi.org/10.1111/j.1600-0528.2012.00719.x

6. Mejía R, Agualimpia C, Torres J, Galán R, Rodríguez W, Minsalud-ASCOFAME. Investigación nacional de Morbilidad Oral. Estudio de recursos humanos para la salud y educación médica en Colombia. Bogotá, Colombia: Ministerio de Salud-Ascofame; 1971. 
7. Ministerio de Salud, Instituto Nacional de Salud, Ascofame (Colombia). Morbilidad Oral. II Estudio Nacional. Bogotá, Colombia: Ministerio de Salud-Instituto Nacional de Salud; 1980.

8. Ministerio de Salud. III Estudio nacional de salud bucal, ENSAB III. Bogotá, Colombia: Ministerio de Salud; 1998.

9. Ministerio de Salud y Protección Social. IV Estudio nacional de salud bucal, ENSAB IV: Para saber cómo estamos y saber qué hacemos. Bogotá, Colombia: Ministerio de Salud y Protección Social; 2013-2014.

10. Ministerio de Salud y Protección Social. Encuesta nacional de salud pública. Bogotá, Colombia: Ministerio de Salud y Protección Social; 2007.

11. Ministerio de Salud y Protección Social. Documento técnico versión 3: Aspectos metodológicos para la construcción de línea base para el Seguimiento a las Metas del Objetivo 3 del Plan Nacional de Salud Pública [internet]. Bogotá, Colombia: Ministerio de Salud y Protección Social; 2010. Disponible en: https://www.minsalud.gov.co/DocumentosyPublicaciones/DOCUMENTOT\%C3\%89CNICOL\%C3\% 8DNEADEBASECONATLASVERSI\%C3\%93N3-ABRIL2010.pdf

12. Departamento Administrativo Nacional de Estadistica. Colombia: Una nación multicultural. Su diversidad étnica. Bogotá, Colombia: DANE; 2005.

13. Heinrich-Weltzien R, Bartsch B, Eick S. Dental caries and microbiota in children with black stain and nondiscoloured dental plaque. Caries Res. 2014; 48(2): 118-25. https://doi.org/10.1159/000353469

14. Guarnizo-Herreno CC, Wehby GL. Explaining racial/ethnic disparities in children's dental health: a decomposition analysis. Am J Public Health. 2012; 102(5): 859-66. https://doi.org/10.2105/AJPH.2011.300 548

15. Freire Mdo C, Reis SC, Figueiredo N, Peres KG, Moreira Rda S, Antunes JL. [Individual and contextual determinants of dental caries in Brazilian 12-year-olds in 2010]. Rev Saude Publica. 2013; 47(Suppl 3): 40-9. h ttp://dx.doi.org/10.1590/S0034-8910.2013047004322

16. Agudelo-Suárez AA, Martínez-Herrera E, Posada-López A, Sánchez-Patino D, Vinas-Sarmiento Y. Ethnicity and self-perceived oral health in Colombia: a cross-sectional analysis. J Immigr Minor Health. 2014; 16(1): 111-8.

17. World Health Organization. Oral health surveys: Basic methods. 5th Ed. Geneva, Switzerland: World Health Organization; 2013.

18. Greene JC, Vermillion JR. The Simplified oral hygiene index. J Am Dent Assoc. 1964; 68: 7-13.

19. Klein H, Palmer CE, Knutson JW. Studies on dental caries. Public Health Rep. 1938; 53: 751-65.

20. Gruebbel AO. A measurement of dental caries prevalence and treatment service for deciduous teeth. J Dent Res. 1944; 23(3): 163-8.

21. Bratthall D. Introducing the Significant Caries Index together with a proposal for a new global oral health goal for 12-year-olds. Int Dent J. 2000; 50(6): 378-84.

22. World Medical Association General Assembly. World Medical Association Declaration of Helsinki: ethical principles for medical research involving human subjects. J Int Bioethique. 2004; 15(1): 124-9.

23. Ministerio de Salud y Protección Social. Resolución 008430 de 1993 (4 de octubre de 1993), por la cual se establecen las normas científicas, técnicas y administrativas para la investigación en salud.

24. Von Elm E, Altman DG, Egger M, Pocock SJ, Gøtzsche PC, Vandenbroucke JP. Declaración de la Iniciativa STROBE (Strengthening the Reporting of Observational studies in Epidemiology): directrices para la comunicación de estudios observacionales. Gac Sanit. 2008; 22(2): 144-50.

25. Meneses-Gómez E, Vivares-Builes A, Posada-Lopez A. Hábitos y prácticas de salud bucal en escolares del área metropolitana del Valle de Aburrá. Rev Colomb Investig Odontol. 2013; 4(12): 187-98. http://dx.doi.org/10. $25063 / 21457735.148$

26. Díaz Cárdenas S, Tirado Amador LR, Madera Anaya MV. Odontología con enfoque en salud familiar. Rev Cubana Salud Públ. 2014; 40(3): 397-405.

27. Ospina D, Herrera Y, Betancur J, Agudelo HB, Posada López A. Higiene bucal en la población de San Francisco Antioquia y sus factores relacionados. Rev Nac Odontol. 2016; 12(22): 23-30. 
28. Abadía Barrero CE. Pobreza y desigualdades sociales: un debate obligatorio en salud oral. Acta Bioeth. 2006; 12(1): 9-22. http://dx.doi.org/10.4067/S1726-569X2006000100002

29. Triana FE, Rivera SV, Soto L, Bedoya A. Estudio de morbilidad oral en niños escolares de una población de indígenas amazónicos. Colomb Med. 2005; 36(4 Supl 3): 26-30.

30. Corchuelo-Ojeda J, Soto-LLanos L, Mambuscay JC. Prevalencia de caries en adolescentes atendidos en la red de salud del Valle del Cauca: alternativas de medición y factores asociados. CES Odontol. 2016; 29(1): 22-32.

31. Agudelo-Suárez AA, Pérez-Escobar JS, Martínez-Herrera E. Related Factors to the use of oral health services amongst population aged 6 to 15 years in Colombia. Int J Odontostomatol. 2014; 8(1): 175-82.

32. UNHCR-ACNUR, ONU. ACNUR y la Oficina de la ONU para los Derechos Humanos solicitan protección para población civil en Chocó [internet]. Bogotá, Colombia: UNHCR-ACNUR, ONU; 12 de mayo del 2016. Disponible en: http://www.acnur.org/noticias/noticia/acnur-y-la-oficina-de-la-onu-para-los-derechos-human os-solicitan-proteccion-para-poblacion-civil-en-choco/

33. González Sevillano PH. ¿ Por qué donde hay tanta riqueza hay tanta pobreza? Evolución de la normatividad y su impacto socio-jurídico en los pobladores de la región pacífico de Colombia: siglo XX. [Con] textos. 2014; 3(10): 43-9.

34. García-Zavaleta C. Equidad y salud bucal. Rev Estomatol Herediana. 2015; 25(2): 85-6.

35. Díaz Cárdenas S, Arrieta Vergara K, Ramos Martínez K. Funcionalidad familiar y caries dental en niños de una institución educativa de Cartagena de Indias. Rev Colomb Investig Odontol [internet]. 2015; 6(16): 41-9. Disponible en: https://www.rcio.org/index.php/rcio/article/view/197.

36. Fisher-Owens SA, Isong IA, Soobader MJ, Gansky SA, Weintraub JA, Platt LJ, Newacheck PW. An examination of racial/ethnic disparities in children's oral health in the United States. J Public Health Dent. 2013; 73(2): 166-74.

37. Lamas Rojas H, Lamas Lara C, Lamas Lara V. Odontología social: desigualdad social y salud. In Crescendo. 2012; 3(1): 139-52. https://doi.org/10.21895/in\%20cres.v3i1.103.

Licencia Creative Commons CC BY 4.0

Cómo citar: Hurtado-Martínez CF, Potes-Urrutia DA, Vásquez-Díaz M, Posada López A, Álvarez-Sánchez LG, Agudelo-Suárez AA. Higiene bucal, caries dental y necesidades de tratamiento en escolares de 5, 7 y 12 años, municipio de Istmina, Chocó, Colombia (2013). Univ Odontol. 2017. jul-dic; 36 (77). http s:// doi.org/10.11144/Javeriana.uo36-77.hbcd 